\title{
Actuator tests for a large deformable membrane mirror
}

\author{
Roger Hamelinck ${ }^{*}{ }^{1}$, Nick Rosielle ${ }^{1}$, Maarten Steinbuch ${ }^{1}$, \\ Rogier Ellenbroek ${ }^{2}$, Michel Verhaegen ${ }^{2}$, Niek Doelman $^{3}$ \\ ${ }^{1}$ Technische Universiteit Eindhoven, Department of Mechanical Engineering, Control Systems \\ Technology, Den Dolech 2, 5600 MB Eindhoven, The Netherlands \\ ${ }^{2}$ Delft University of Technology, Center for Systems and Control, \\ Mekelweg 2, 2628 CD Delft, The Netherlands \\ ${ }^{3}$ TNO Science and Industry, Opto-Mechanical Instrumentation, \\ Stieltjesweg 1, 2628 CK Delft, The Netherlands
}

\begin{abstract}
In the design of a large adaptive deformable membrane mirror, variable reluctance actuators are used. These consist of a closed magnetic circuit in which a strong permanent magnet provides a static magnetic force on a ferromagnetic core which is suspended in a membrane. By applying a current through the coil which is situated around the magnet, this force is influenced, providing movement of the ferromagnetic core. This movement is transferred via a rod imposing the out-ofplane displacements in the reflective deformable membrane. In the actuator design a match is made between the negative stiffness of the magnet and the positive stiffness of the membrane suspension. If the locality of the influence functions, mirror modes as well as force and power dissipation are taken into account, a resonance frequency of $1500 \mathrm{~Hz}$ and an overall stiffness of $1000 \mathrm{~N} / \mathrm{m}$ for the actuators is needed. The actuators are fabricated and the dynamic response tested in a dedicated setup. The Bode diagram shows a first eigenfrequency of $950 \mathrm{~Hz}$. This is due to a lower magnetic force than expected. A Helmholtz coil setup was designed to measure the differences in a large set of permanent magnets. With the same setup the $2^{\text {nd }}$ quadrant of the $\mathrm{B}-\mathrm{H}$ curve is reconstructed by stacking of the magnets and using the demagnetization factor. It is shown that the values for $\mathrm{H}_{\mathrm{c}}$ and $\mathrm{B}_{\mathrm{r}}$ of the magnets are indeed lower than the values used for the initial design. New actuators, with increased magnet thickness, are designed and currently fabricated.
\end{abstract}

Keywords: electromagnetic, actuator, deformable, mirror, Helmholtz, coil

\section{INTRODUCTION}

For challenging adaptive optics applications on existing telescopes as well as future extremely large telescopes (ELT's) the further development of deformable mirror technology is essential. The requirements for deformable mirrors for these systems become more demanding, in particular the need for significantly more actuators than currently available on the market, poses a significant technical challenge. Deformable mirrors are required to have thousands of actuators, to position the mirror surface with nm accuracy and to operate at extremely high bandwidths. This in combination with low total power dissipation and reasonable cost. A design that potentially fulfils these future requirements was presented in [1] and [2]. In [1] the working principle and design, and presents the first prototypes of the electromagnetic actuators used as well as a 61 actuator grid to be used as a standard building block for large arrays of actuators.

* r.f.m.m.hamelinck@tue.nl; phone ++31 (0)40 247 4580; fax ++31 (0)40 246 1418; www.wtb.tue.nl 
A companion paper at this conference [6272-123], will discuss a fully distributed controller framework that addresses the problems involved with controlling large amounts of actuators with high bandwidth.

This paper focuses on the dynamic measurements of the actuators. These measurements are compared with the design specifications. De differences found will be explained and validated by measurement of the permanent magnetic properties of the magnets used. For these measurements a dedicated Helmholtz coil test setup is designed and fabricated.

\section{THE ACTUATOR DESIGN}

\subsection{The variable reluctance actuator}

The actuator design is extensively described in [1]. A brief overview of the actuator design will be given here. Figure 2.1 shows the schematic of upper layers in the deformable mirror. Three actuators are shown, situated in a common base plate. Each actuator is connected via a rod with the deformable membrane which acts as the reflective, correcting element. Figure 2.2 shows the cross-section of one actuator.

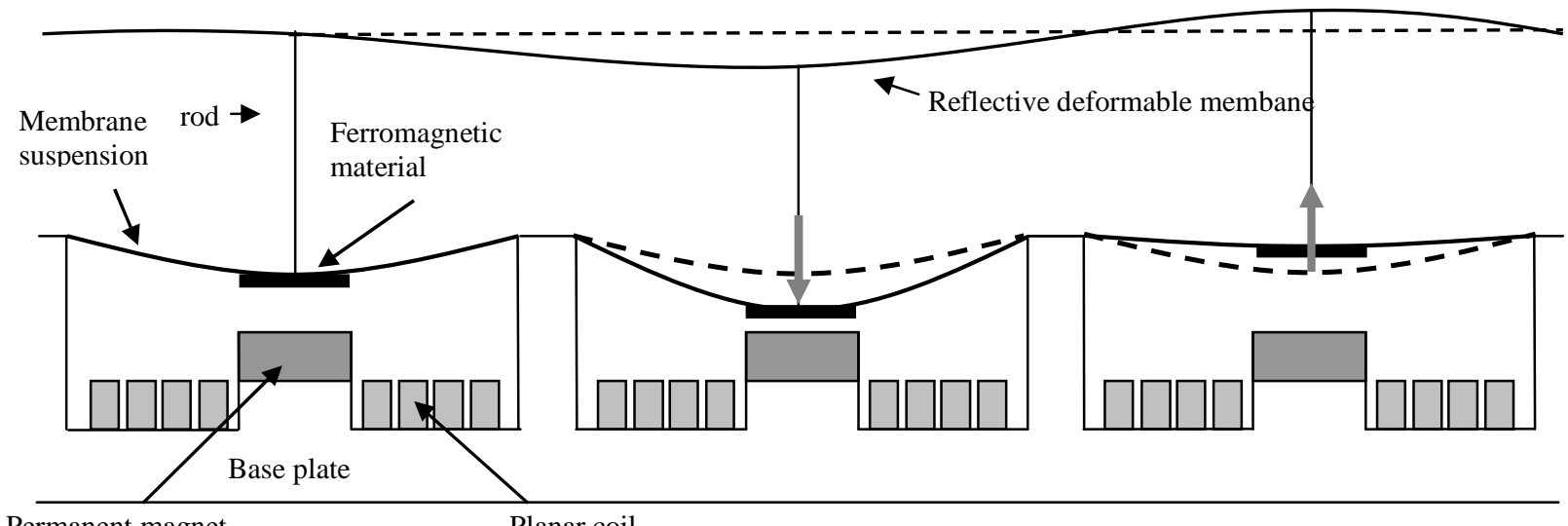

Permanent magnet

Planar coil

Figure 2.1: Schematic of the base plate with three actuators and the connection via rods with the reflective membrane surface.

Each actuator consists of a permanent magnet (1), a surrounding coil (2), an airgap (3), a ferromagnetic moving core (4), a membrane suspension (5), a second airgap (6), and a base plate (7). The magnetic flux crosses the first airgap, through the ferromagnetic core, crosses the second airgap to the pole shoes (8) and returns via the base plate back to the other pole of the permanent magnet.

The attraction force of the permanent magnet on the ferromagnetic core gives a static deflection of the membrane suspension. The stable equilibrium position is determined by the (negative) magnetic force and the

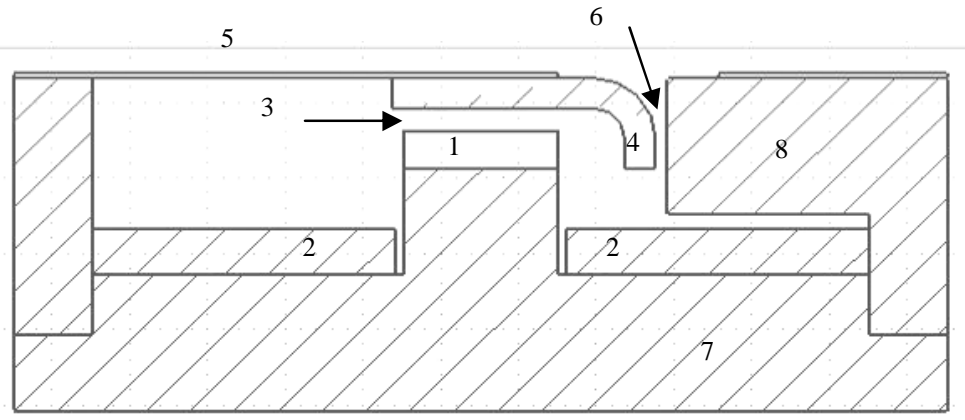
(positive) restoring force of the membrane suspension. The magnetic force, and thereby the deflection of the membrane, can be decreased as well as increased by a current through the surrounding planar coil. This results in a movement of the ferromagnetic core and provides each actuator with the necessary stroke to impose the mirror's deformation. Figure 2.3 show pictures of the first actuators. The layer based design makes the actuators easily producible in an array. Figure 2.4 the base plate for a 61-actuator grid. The grid serves as a standard building block for large arrays of actuators. 


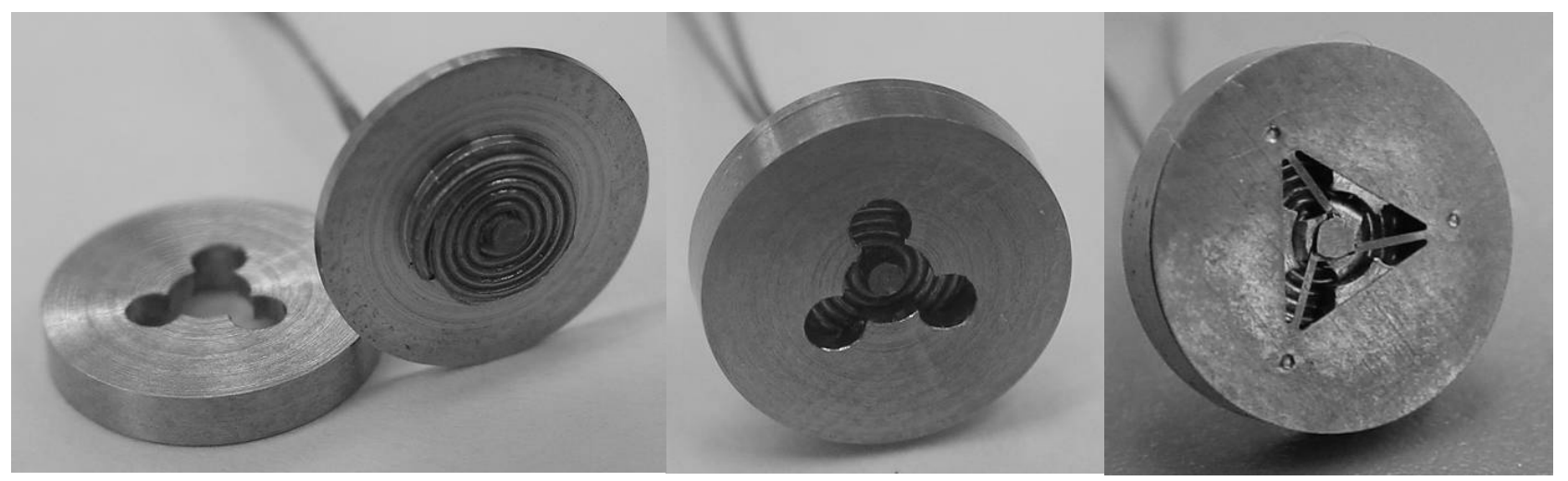

Figure 2.3: The different parts of the first actuator and the assembled actuator on the right.

This base plate will be covered by the $30 \mu \mathrm{m}$ thick titanium foil, forming the suspension for the ferromagnetic cores. From the backside, subassemblies with the coils and permanent magnets can be plugged in. It is possible to arrange the actuators in every desirable grid; hexagonal, orthogonal or polar. The actuator arrays will be connected to the support structure as shown in [2].

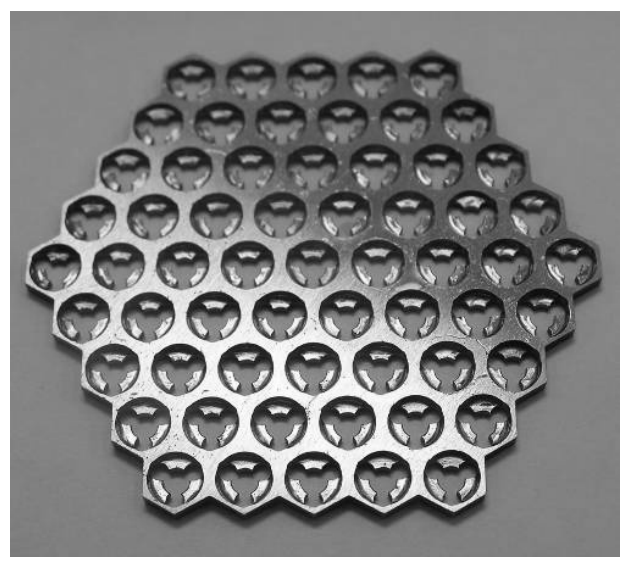

Figure 2.4: The backside of the base plate for a 61-actuator array (left). The cad drawing shows a detail of the filled actuator grid and mirror on top (right).

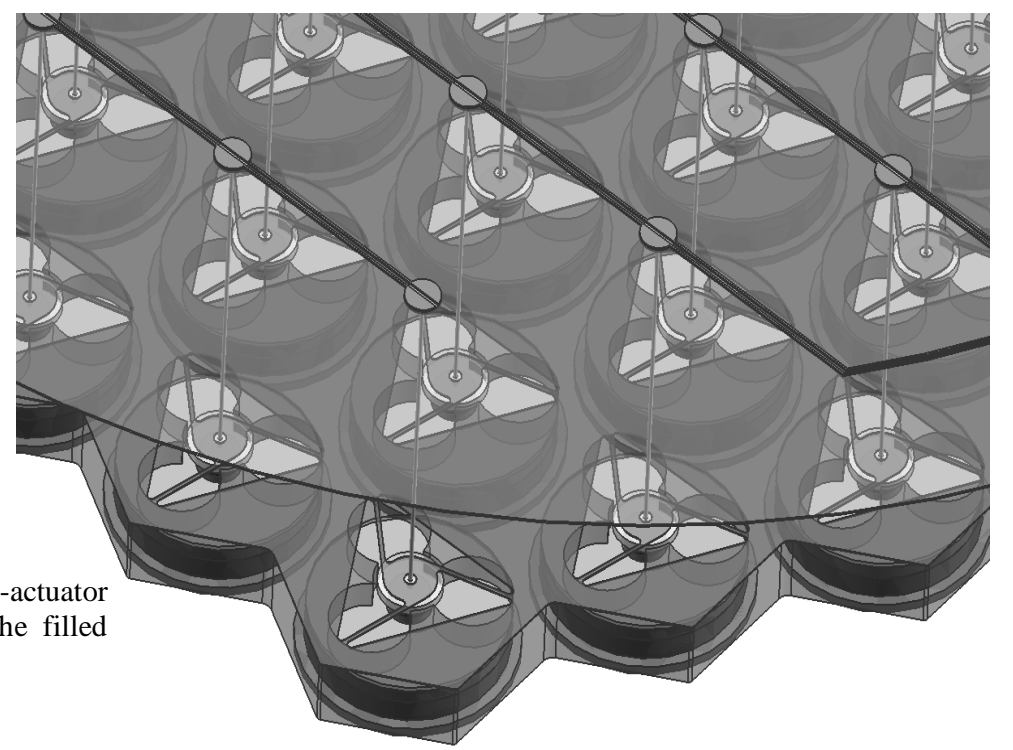

\subsection{Actuator analysis}

A very important parameter of the actuator is its stiffness. It determines the influence function, the local and global resonance frequencies of the mirror and the force needed for a displacement, and thereby the power dissipation. Effects are described in [2,3]. An increase in actuator stiffness will result in a more local influence function and higher resonance frequencies, but also lead to increased actuator forces and power dissipation.

The stiffness of the actuator is determined by the (positive) stiffness of the membrane suspension for the ferromagnetic core and the (negative) stiffness of the permanent magnet.

The electromagnetic force $\left(\mathrm{F}_{\mathrm{m}}\right)$ acting on the ferromagnetic core is calculated with an electrical equivalent circuit analysis and given by [1]:

$F_{m}=-\frac{1}{2 \mu_{0} A_{m}}\left(\frac{H_{c} d_{m}+N I}{R_{e q}}\right)^{2}$ 
In which $\mu_{0}$ is the permeability of vacuum $\left[4 \pi \cdot 10^{-7} \mathrm{Tm} / \mathrm{A}\right], A_{m}$ is the permanent magnet area $\left[\mathrm{m}^{2}\right], H_{c}$ is the coercive force of the permanent magnet $[\mathrm{A} / \mathrm{m}], d_{m}$ is the thickness of the magnet $[\mathrm{m}], N$ is number of windings in the coil, $I$ is the current through the coil [A] and $R_{e q}$ is the equivalent reluctance of the magnetic circuit $\left[\mathrm{H}^{-1}\right]$.

The non-linear restoring force of the membrane suspension for the ferromagnetic core can be approximated by [4,5]:

$F_{s}=A \frac{E t^{3}}{r^{2}} z+B \frac{E t}{r^{2}} z^{3}$

In which $E$ is the Young's modulus $\left[\mathrm{N} / \mathrm{m}^{2}\right], t$ is the thickness $[\mathrm{m}]$ and $r$ is the radius $[\mathrm{m}]$ of the actuator membrane. $z$ is the membrane deflection $[\mathrm{m}]$. The coefficients A and B depend on the boundary conditions of the plate. A linear and a nonlinear term can be distinguished, representing the bending stiffness and the stiffness from the in plane stretching of the membrane as it occurs with larger displacements.

The actuators are designed to have a force equilibrium at an initial airgap of $75 \mu \mathrm{m}$ and to have a stiffness 'match' between the membrane suspension and magnet that results in $1000 \mathrm{~N} / \mathrm{m}$ overall stiffness. With the moving mass of the actuator, it gives a first resonance frequency of $1.5 \mathrm{kHz}$. If the mass of the reflective membrane is placed on top, this reduces to approximately $1 \mathrm{kHz}$. This result is independent of the mirror size; if the mirror 'grows' not only mass is added but also stiffness [3].

\section{DYNAMIC MEASUREMENTS OF THE ACTUATOR}

The dynamic properties of the actuators shown in figure 2.3 were measured with a test setup [6] as shown in figure 3.1 . The actuator is placed in a container and placed in front of an optical displacement sensor [7]. The actuator is electrically connected to a signal generator. White noise current with frequencies up to $1800 \mathrm{~Hz}$ was applied to the actuator. For calibration purposes the optical displacement sensor can be moved in axial direction on a parallel guiding mechanism. This is done with a micro spindle connected to a lever and the elastic guiding mechanism. The lever and parallel guiding mechanism are monolithic, thereby avoiding friction, play and hysteresis.

A set of input-output data was obtained by measuring the displacement of the ferromagnetic core in the actuator due to the white noise current signal applied to the actuator. The Bode-diagram is shown in figure 3.2. With this data, a continuous time mass spring damper model was estimated using frequency domain identification. The moving

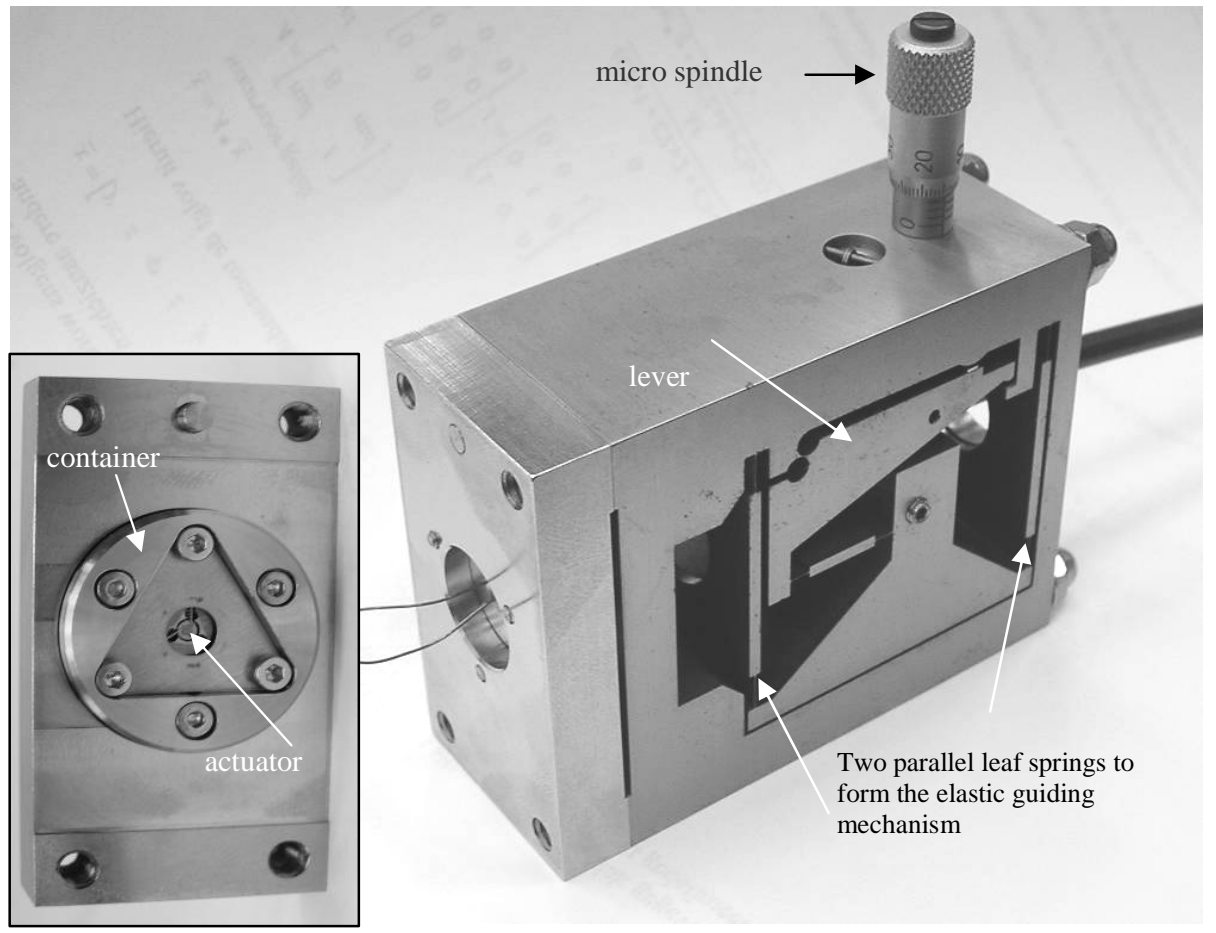

Figure 3.1: The actuator placed in the measurement setup to measure the dynamic properties of the actuator

mass $m$, the stiffness $c$ and damping $b$ of the actuator are identified from the model parameters:

$m=9.9 \cdot 10^{-3}[\mathrm{~g}], \quad b=1.4 \cdot 10^{-3}[\mathrm{Ns} / \mathrm{m}], \quad c=330[\mathrm{~N} / \mathrm{m}]$ 
Figure 3.2 shows a clear resonance peak around 950 $\mathrm{Hz}$. This was designed to be $1500 \mathrm{~Hz}$. Since the moving mass is well known, the difference is explained to be caused by lower actuator stiffness. The measured stiffness is $330 \mathrm{~N} / \mathrm{m}$ instead of the designed $1000 \mathrm{~N} / \mathrm{m}$. This mechanical stiffness is, as explained in section 2.1 and 2.2, a sum of the positive stiffness of the membrane suspension of the moving ferromagnetic core and the negative stiffness of the permanent magnet. The non-linear stiffness of the membrane suspension is measured and described in [1]. These measurements were in good agreement with the non-linear stiffness from the non-linear FEM calculations [1].

A possible explanation for the differences in resonance can be found in the permanent magnets. If the permanent magnets are weaker than expected, the magnetic attraction to the ferromagnetic core, which is static load on the membrane suspension, will be lower. Since the non-linear stiffness of the membrane depends on this load it will operate in a lower stiffness regime.

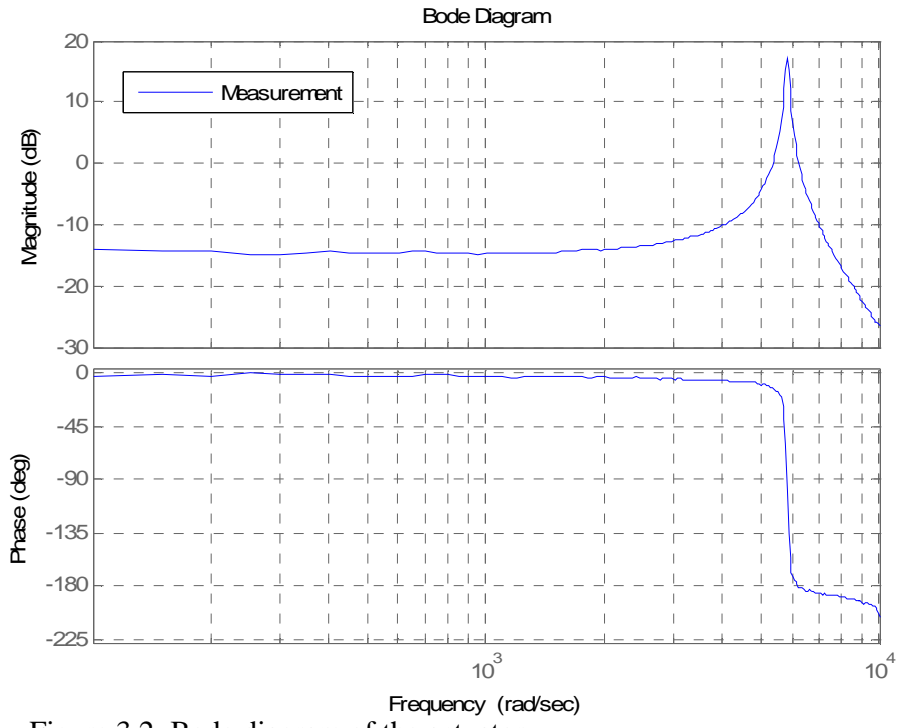

Figure 3.2: Bode diagram of the actuator

Since there is reason to suspect that the magnetic properties of the magnets differ from the assumed properties measurements were taken. To measure the magnetic properties a Helmholtz coil in combination with a commercial fluxmeter was used.

Advantages of such a test setup are:

- the measurement is performed fast and reasonably accurate,

- no possibly destructive external field is applied to the magnets,

- $\quad$ the measurement system and measurements are low cost

The test setup is used to measure differences within a set of magnets and to reconstruct the $2^{\text {nd }}$ quadrant of the B-H curve of the permanent magnets using measurements.

\section{MAGNETIC MEASUREMENTS WITH A HELMHOLTZ COIL}

\subsection{Measurement principle of a Helmholtz coil}

In figure 4.1, the Helmholtz coil measurement setup is shown schematically. The Helmholtz coil consists of a pair of identical circular coils on a common axis. If the coils are connected in series and current flows through the coils in the same direction, a uniform magnetic field is produced at the center. With the BiotSavart law, the magnetic field produced by a current loop can be calculated. The magnetic field from the two coils of the Helmholtz coil arrangement can be obtained by superimposing the two constituent fields. The primary component of the uniform magnetic field is parallel to the axes of the two coils. This uniform field is the result of the addition of the two field components parallel to the axes of the two coils and the difference between the components perpendicular to the axes.

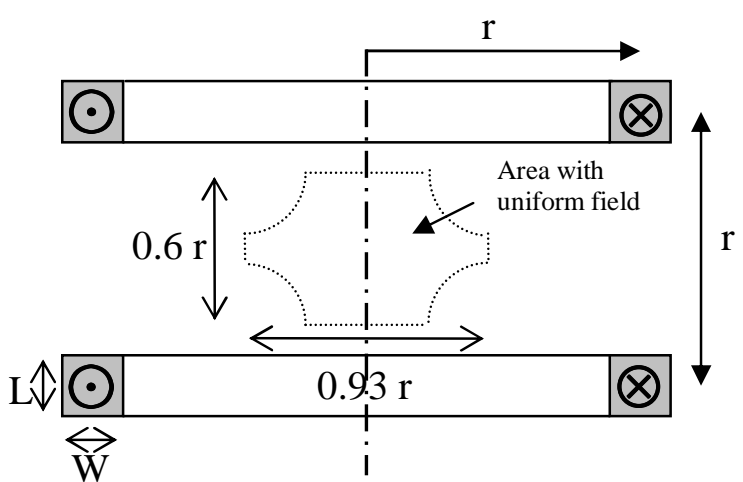

Figure 4.1: Schematic of a Helmholtz coil 
For a given coil radius, the optimal coil separation can be calculated needed for the largest uniform central field. This separation is equal to the radius of the coils. Furthermore, in order to get a homogeneous field for coils with finite dimensions, the coil's cross-section should be according to [8]:

$\frac{W}{L}=\sqrt{\frac{36}{31}}$

The area with a uniform field ( $<1 \%$ deviation) is also given in figure 4.1.

A Helmholtz coil can not only be used to produce a uniform magnetic field, but also, if coupled to an integrating voltmeter or fluxmeter, to measure the magnetic dipole moment of a permanent magnet placed in that region [9]. With a Helmholtz coil the magnetic dipole moment at the actual operating point of the permanent magnet is measured. The measured magnetic dipole moment depends on the magnet volume, shape and its relative permeability. The magnetic moment represents the product of the pole strength and magnetic length of the sample. The pole strength is a measure of total flux at the pole and the magnetic length is not the same as physical length of the magnet. The actual magnetic length varies from 0.7 times the physical length in long and/or low coercivity magnets to nearly the physical length in short and/or high coercivity magnets and varies with the level of magnetization.

By reciprocity, the coil flux caused by the dipole will not depend on the position in the area of uniform field as described above. If the magnet is placed closer to one coil, that coil is cut by more flux and the other coil by less, but the total flux seen by the two coils remains constant. By Faraday's law it follows that the voltage generated is proportional with the rate of the change of the flux in the loop. Magnetic flux is hereby defined as the product of the magnitude of the magnetic field and the area of the loop. By inserting or extracting a permanent magnet, the voltage induced can be integrated as a measure for the dipole moment. If the inserted magnet is rotated over 180 degrees on an axis perpendicular to the coil axis, the magnetic dipole moment as measured is doubled.

A turn at the centre of the cross section of each of the two coils will enclose a specific flux when a magnetic dipole, with $\mathrm{m}_{\mathrm{z}}$ as a dipole moment is inserted in the centre of the coil. This flux is given by [9]:

$\varphi=\frac{\mu_{0} m_{z} N}{r} \frac{2}{5} \sqrt{\frac{4}{5}}$

For a coil with $\mathrm{N}$ turns and a finite cross section the coupled flux is [9]:

$\varphi=\frac{\mu_{0} m_{z} N}{r} \frac{2}{5} \sqrt{\frac{4}{5}}\left\{1-\frac{1}{60}\left(\frac{w}{r}\right)^{2}\right\}$

$=k_{H} m_{z}$

This constant $k_{H}$ is therefore linked to the Helmholtz coil geometry.

\subsection{Reconstructing the $2^{\text {nd }}$ quadrant of the $B-H$ curve}

Because the magnets used in the actuator can be stacked on top of each other, it is possible to reconstruct a significant part of this B-H curve with the Helmholtz coil test setup [9].

The $2^{\text {nd }}$ quadrant of the B-H loop as in figure 4.2 can be described by:

$B=\mu_{0} H+\mu_{0} M(H)$

$=\mu_{0} \mu_{r} H+B_{r}$

Where $B$ is the magnetic induction [T], $B_{r}$ is the magnetic remanence [T], $\mu_{0}$ is the permeability in vacuum $\left.\left[4 \pi \cdot 10^{-7} \mathrm{Tm} / \mathrm{A}\right)\right], \mu_{r}$ is the relative permeability, $M$ is the magnetization $[\mathrm{A} / \mathrm{m}]$ and $H$ is the magnetic field strength $[\mathrm{A} / \mathrm{m}]$.

The average magnetization $M(H)$ per unit volume of the measured (stack) permanent magnets can be calculated by:

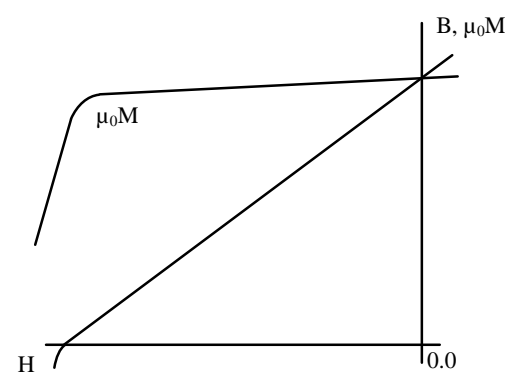

Figure 4.2: B-H curve of a permanent magnet 
$M(H)=\frac{m_{z}(H)}{V}$

This measured value is lower than the value of $M_{0}$ because $M(H)$ is dependent on the internal field in the magnet:

$M=\frac{M_{0}}{\mu_{r}}=\frac{\mu_{0}-1}{\mu_{0} \mu_{r}} B$

The average value of $B$ inside the magnet in air depends only on the magnet shape. The internal B-value increases with the relative length in the magnetization direction.

To obtain the value of $B$ in (4.4) the value for $H$ and $M(H)$ is needed. It is possible to obtain the value of $B$ by the introduction of the demagnetization factor $\mathrm{N}_{\mathrm{m}}$.

$H=-N_{m} M$

The value of $\mathrm{N}_{\mathrm{m}}$ is between 0 and 1 and depends only on the shape of the magnet [10].

The process to get from the measurements with the Helmholtz coil to a reconstructed B-H curve is:

a) Measuring the magnetic dipole moment $m_{z}$ of stacks of the permanent magnets (e.g. 1,2,3,..., 16 magnets).

b) Calculate the average value of $M(H)$ per unit volume.

c) Determining the demagnetization factor for each of the stacks, depending on the diameter and height.

d) Calculate the value of the magnetic field strength $H$.

e) Calculate the average value of the flux density $B$.

The found values can be plotted in the second quadrant of the B-H curve.

\subsection{Design of the Helmholtz coil setup}

A dedicated Helmholtz setup is fabricated to measure the small magnets used in the actuators $(\varnothing 1 \mathrm{x} 0.3 \mathrm{~mm})$. The mean diameter of the coils is $15 \mathrm{~mm}$. Each coil has 580 windings with 50 micrometer copper wire. The coil constant for the conversion between measured flux $[\mathrm{Vs}]$ and magnetization $\left[\mathrm{Am}^{2}\right]$ is $6.87 \mathrm{e}-2$.

$\varphi=\frac{\mu_{0} m_{z} N}{r} \frac{2}{5} \sqrt{\frac{4}{5}}\left\{1-\frac{1}{60}\left(\frac{w}{r}\right)^{2}\right\}$

$=6.87 \cdot 10^{-2} m_{z}$

The Helmholtz coil setup has a rotation table into which the magnets can be inserted and two stops to rotate the table over 180 degrees. (Figure 4.3)

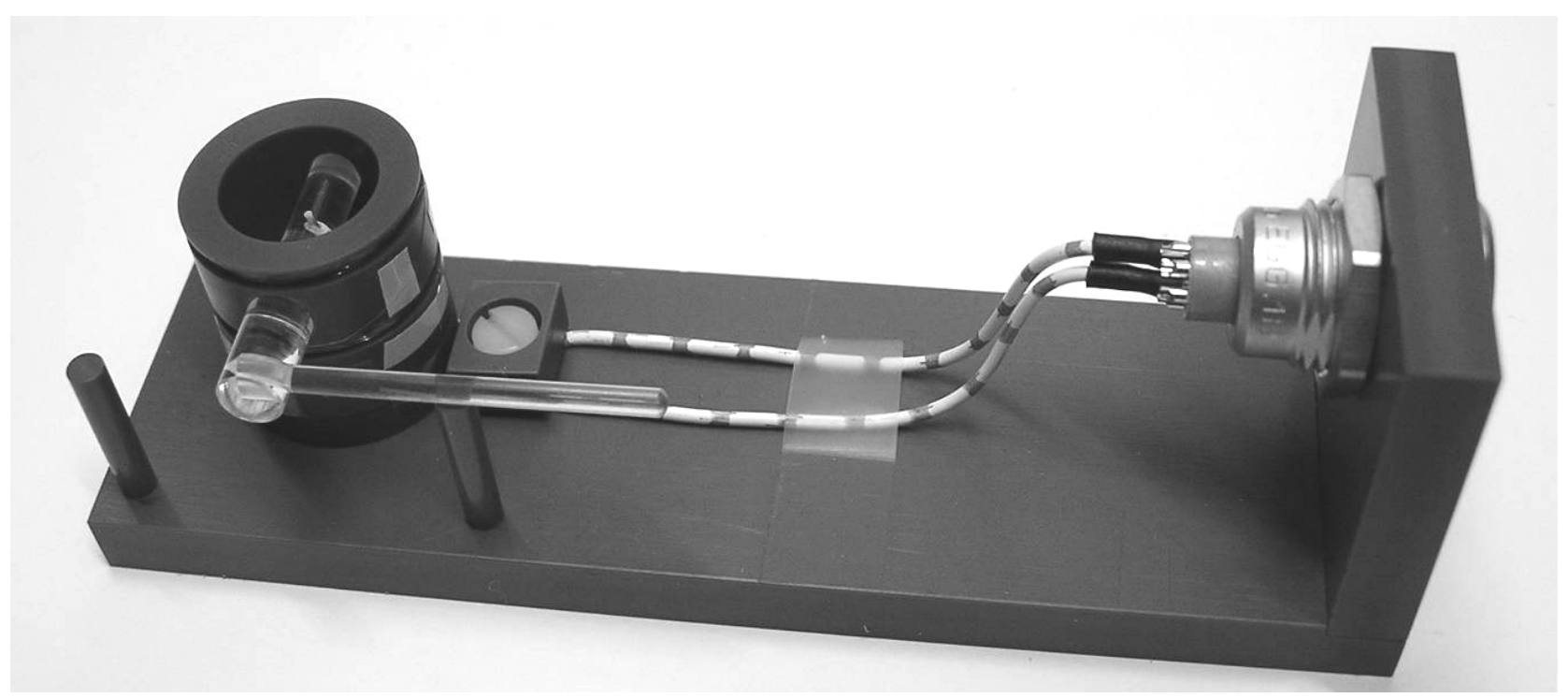



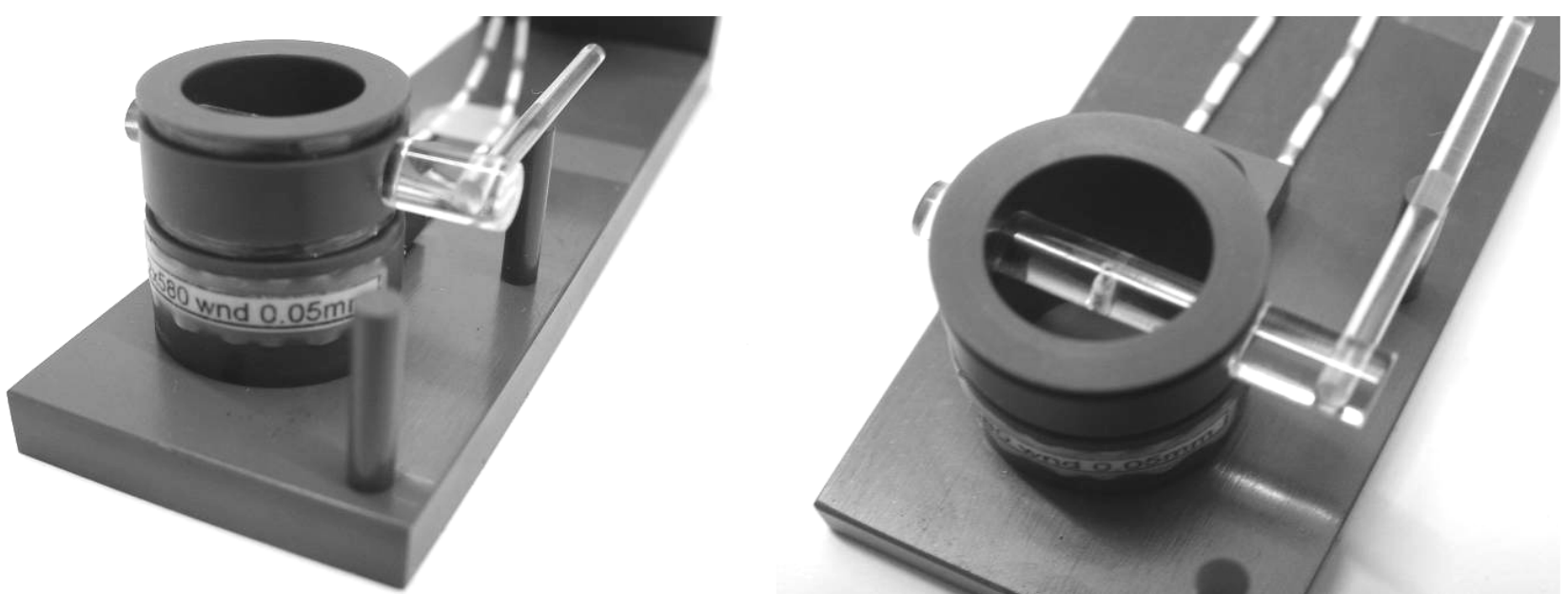

Figure 4.3: The Helmholtz coil test set up. The two coils $(\varnothing 15 \mathrm{~mm})$ and the rotation table with the two stops to rotate the table with magnet over 180 degrees are visible.

\subsection{Measurement of the differences in magnetic properties}

A set of $200 \mathrm{SmCo}_{5}$ magnets with dimensions $\emptyset 1.0 \mathrm{x} 0.3 \mathrm{~mm}$ were measured. The histogram of the flux measurements with the designed Helmholtz coil and commercial fluxmeter is given in figure 4.4. The figure shows that one of the magnets is probably not well magnetized. All other magnets show small differences: the mean value for the integrated voltage is $9.6 \mathrm{Vs}$ and the variance is $0.05 \mathrm{~V}^{2} \mathrm{~s}^{2}$.

\subsection{Reconstruction of the B-H curve}

A set of magnets with the mean measured value is taken for the reconstruction of the $2^{\text {nd }}$ quadrant of the B-H curve. The steps as described in section 4.2 to get from the flux measurements with the Helmholtz coil to a reconstructed B-H curve are taken. The result is shown in figure 4.5. From the supplier of the permanent magnets the values for $\mathrm{H}_{\mathrm{c}}=676 \pm 40 \mathrm{kA} / \mathrm{m}$ and $\mathrm{B}_{\mathrm{r}}=1.05 \pm 0.05 \mathrm{~T}$ are plotted.

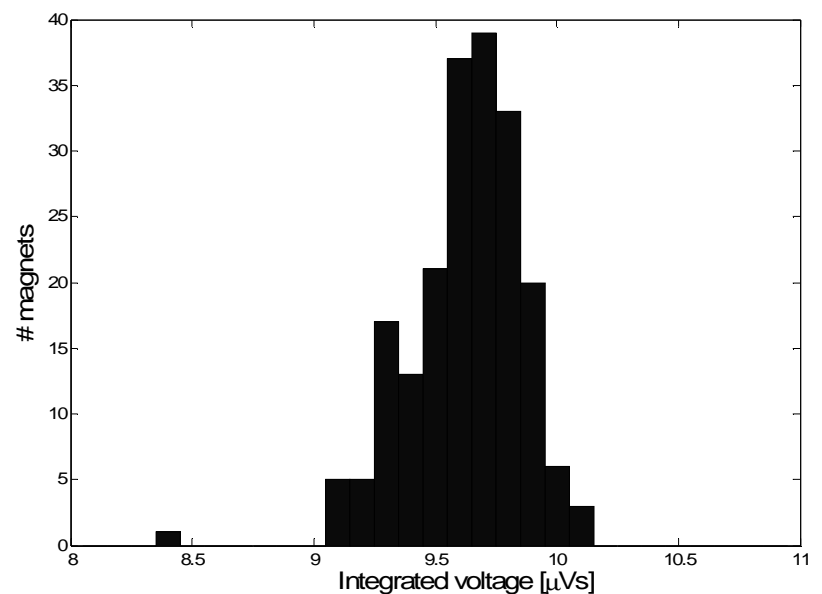

Figure 4.4: The histogram of $200 \mathrm{SmCo}_{5}$ magnets $(\varnothing 1.0 \mathrm{x} 0.3 \mathrm{~mm})$.

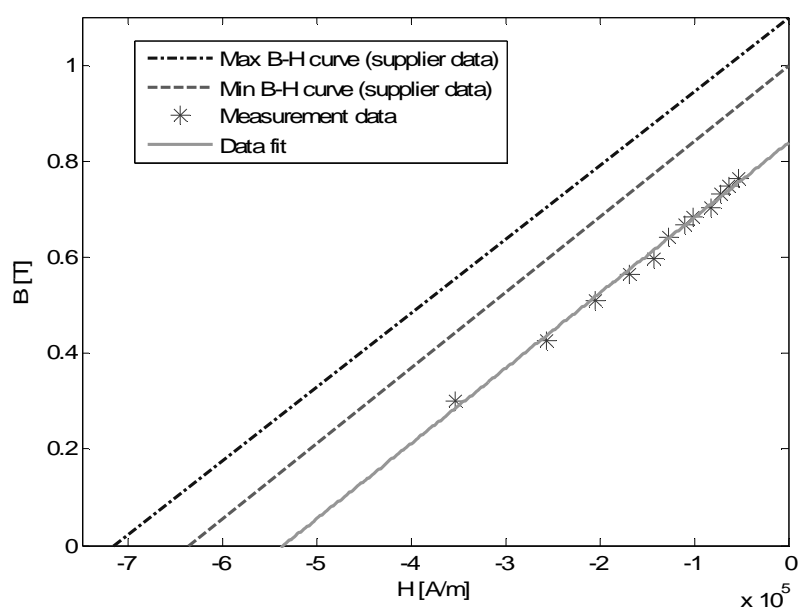

Figure 4.5: The $2^{\text {nd }}$ quadrant of the $\mathrm{B}-\mathrm{H}$ curve for the $\mathrm{SmCo}_{5}$ magnets. The supplier's upper en lower boundary as well as the measurement data and fit are shown. 


\subsection{Conclusions on the magnetic measurements}

From figure 4.5 it is clear that the magnetic properties of the measured permanent magnets are different from the expected values. Both $\mathrm{H}_{\mathrm{c}}$ and $\mathrm{B}_{\mathrm{r}}$ are smaller, resulting in a lower magnetic force on the ferromagnetic core. The assumption that the permanent magnets would be the reason for the lower resonance frequency in figure 3.2 is shown to be correct. In order to get the same magnetic force in the actuators, the thickness of the permanent magnet used in the actuator is increased.

New actuators with the increased magnet thickness are designed en currently fabricated.

\section{CONCLUSION}

In the design of a large adaptive deformable membrane mirror, variable reluctance actuators are used. These consist of a closed magnetic circuit in which a strong permanent magnet provides a static magnetic force on a ferromagnetic core which is suspended in a membrane. By applying a current through the coil which is situated around the magnet, this force is influenced, providing movement of the ferromagnetic core in its suspension. This movement is transferred via a rod imposing the out-of-plane displacements in the reflective deformable membrane. In the actuator design a match is made between the negative stiffness of the magnet and the positive stiffness of the membrane suspension where the ferromagnetic core is connected to. If the locality of the influence functions, local and global modes as well as force and resulting power dissipation are taken into account, a resonance frequency of $1500 \mathrm{~Hz}$ and an overall stiffness of $1000 \mathrm{~N} / \mathrm{m}$ for the actuators is needed. The actuators are fabricated and the dynamic response tested in a dedicated setup using a non contact optical displacement sensor. A Bode diagram of the actuator is measured. The Bode diagram shows a first resonance frequency of $950 \mathrm{~Hz}$ instead of the designed $1500 \mathrm{~Hz}$. This is due to a lower magnetic force than expected. A Helmholtz coil setup was designed to measure the differences in a large set of permanent magnets. With the same setup the $2^{\text {nd }}$ quadrant of the B-H curve is reconstructed by stacking of the magnets and using the demagnetization factor. It is shown that the values for $\mathrm{H}_{\mathrm{c}}$ and $\mathrm{B}_{\mathrm{r}}$ of the magnets are indeed lower than the values used for the initial design. New actuators, with increased magnet thickness, are designed and currently fabricated.

\section{ACKNOWLEDGEMENTS}

The design, realization and testing of this high actuator density mirror is a cooperation of the Technical University of Eindhoven, the opto-mechanical instrumentation department of TNO Science and Industry and the Technical University of Delft. The work done is supported by SenterNovem, the Innovative Research Project (IOP) Precision Technology.

\section{REFERENCES}

1. R. Hamelinck, N. Rosielle, M. Steinbuch, N. Doelman, "Large adaptive deformable membrane mirror with high actuator density: design and first prototypes", $5^{\text {th }}$ International Workshop on Adaptive Optics for Industry and Medicine, SPIE Vol 6018, Beijing, China, 2006

2. R. Hamelinck, N. Rosielle, P. Kappelhof, B. Snijders, M. Steinbuch, "Large adaptive deformable membrane mirror with high actuator density”, SPIE Proc. Vol. 5490, pp. 1482-1492, 2004

3. R. Ellenbroek, M. Verhaegen. N. Doelman, R. Hamelinck, N. Rosielle, M. Steinbuch, "Distributed control in adaptive optics: DM and turbulence modeling”, SPIE Proc. Vol 6272, 2006

4. Stephen P. Timoshenko, S. Woinowsky-Krieger, "Theory of plates and shells", $2^{\text {nd }}$ ed, McGraw-Hill, London, 1970

5. C. Aerts, "Optimizing and implementing leafsprings for the reluctance motor", Technical University Eindhoven, 2005

6. E.J.A. Manders, Design of a device to measure the out-of-plane, nonlinear stiffness of circular membranes, Technische Universiteit Eindhoven, Eindhoven, 2004

7. Homepage Philtec sensors, http://www.philtec.com

8. J.C. Maxwell, "A treatise on electricity \& magnetism', Vol. 2 pp 356-357, Dover, New York, 1954

9. J.C.Compter, .L.F. van der Veen, "Magnetic analysis with a Helmholz coil set", IEE Proc. Sci Meas. Technol. Vol 150, No. 4, July 2003

10. Du-Xing Chen, James A. Brug, "Demagnetizing Factors for Cylinders", Vol. 27. No 4, IEEE Transactions on magnetics, July 1991. 